\title{
EXTRAGALACTIC DARK MATTER AND DIRECT DETECTION EXPERIMENTS
}

\author{
A. N. BAUSHEV \\ DESY, D-15738 Zeuthen, Germany; baushev@ gmail.com \\ and \\ Institut für Physik und Astronomie, Universität Potsdam, D-14476 Potsdam-Golm, Germany \\ Received 2013 January 31; accepted 2013 May 8; published 2013 June 24
}

\begin{abstract}
Recent astronomical data strongly suggest that a significant part of the dark matter content of the Local Group and Virgo Supercluster is not incorporated into the galaxy halos and forms diffuse components of these galaxy clusters. A portion of the particles from these components may penetrate the Milky Way and make an extragalactic contribution to the total dark matter containment of our Galaxy. We find that the particles of the diffuse component of the Local Group are apt to contribute $\sim 12 \%$ to the total dark matter density near Earth. The particles of the extragalactic dark matter stand out because of their high speed $\left(\sim 600 \mathrm{~km} \mathrm{~s}^{-1}\right)$, i.e., they are much faster than the galactic dark matter. In addition, their speed distribution is very narrow $\left(\sim 20 \mathrm{~km} \mathrm{~s}^{-1}\right)$. The particles have an isotropic velocity distribution (perhaps, in contrast to the galactic dark matter). The extragalactic dark matter should provide a significant contribution to the direct detection signal. If the detector is sensitive only to the fast particles $\left(v>450 \mathrm{~km} \mathrm{~s}^{-1}\right)$, then the signal may even dominate. The density of other possible types of the extragalactic dark matter (for instance, of the diffuse component of the Virgo Supercluster) should be relatively small and comparable with the average dark matter density of the universe. However, these particles can generate anomaly high-energy collisions in direct dark matter detectors.
\end{abstract}

Key words: astroparticle physics - dark matter - elementary particles - Local Group

Online-only material: color figure

\section{INTRODUCTION}

It is widely believed that all the dark matter particles (DMPs), which a terrestrial observer can detect, belong to the Milky Way. The main aim of this paper is to dispute this assertion and to show that a remarkable fraction of DMPs detected from Earth likely do not belong to our Galaxy. Although their density is relatively small, as compared with the total dark matter density, their impact on the direct detection signal may even dominate because of the high speeds of the particles.

According to the modern cosmological notion, the halos of giant galaxies, such as the Milky Way or Andromeda, are regions of local dark matter overdensity rather than isolated islands. Indeed, the Local Group, along with the halos of large and dwarf galaxies, contains a significant fraction of dark matter that is not bound in the galaxies and presumably forms a large envelope of the Local Group (Binney \& Tremaine 2008). A significant part of the dark matter of the Virgo Supercluster is also not localized in halos and is probably distributed more or less homogeneously over the volume of the Supercluster (Makarov \& Karachentsev 2011). Some part of this diffuse dark matter (preeminently from the Local Group envelope) penetrates into the central region of our Galaxy and contributes to the direct detection signal, which can even dominate under certain conditions: as we will see, the density fraction of the extragalactic dark matter is relatively small $(\sim 12 \%)$, however, its particles should have extremely high speeds, close to the escape velocity $\left(\sim 600 \mathrm{~km} \mathrm{~s}^{-1}\right)$ or even higher. It sets off the extragalactic particles from the halo DMPs with a much lower average speed. The direct detection signal produced by this component should also have some other characteristic features that will be discussed below.

\section{THE DARK MATTER ENVELOPE OF THE LOCAL GROUP}

Unfortunately, the total mass, distribution, and dynamical properties of the extragalactic dark matter environment are poorly known. Therefore, we have to manage with rough estimates of its content near the solar system.

The Local Group consists of two very massive galaxies (the Milky Way and Andromeda galaxy M31), the less massive Triangulum galaxy M33, and a host of dwarf galaxies. It seems reasonable to say that the Local Group contains a massive diffused dark matter component as well (see Binney \& Tremaine 2008; Makarov \& Karachentsev 2011; Kahn \& Woltjer 1959; Cox \& Loeb 2008 and references therein). Unfortunately, some parameters of the system have not been measured with adequate accuracy. We accept the following values in this paper: the radius of the solar system orbit $l_{\odot}=8 \mathrm{kpc}$, the Milky Way mass $M_{\mathrm{MW}}=10^{12} M_{\odot}$, the Andromeda galaxy mass $M_{31}=1.6 \times 10^{12} M_{\odot}$, and the distance between them $d=750 \mathrm{kpc}$ (Cox \& Loeb 2008). The tangential components of the velocities of even some massive members of the Local Group are also not quite explored, and we know almost nothing about the distribution and dynamical parameters of the diffused component; thus, the investigation of its motion in the complex gravitational field of several bodies is quite a difficult and underdefined task. However, our goal is much simpler: we aim to model just the process of the envelope dark matter penetration toward the solar system. Taking into account all the abovementioned uncertainties, we construct a simple toy model that does not claim to describe all properties of the Local Group, but which allows us, however, to estimate the density and the velocity distribution of the extragalactic dark matter near the solar system.

Let us consider the following model: the system is stationary and spherically symmetric. Then, each particle can be characterized by its specific angular momentum $\mu \equiv[\boldsymbol{v} \times \boldsymbol{r}]$ and the maximum radius $r_{0}$ from the center that the particle may reach. Both these quantities remain constant in such a system, and gravitational potential $\phi(r)$ depends only on $r . r_{0}$ of the particles belonging to the envelope lies in some interval $\left[r_{\mathrm{in}}, r_{\mathrm{out}}\right]$. We accept $r_{\text {in }}=300 \mathrm{kpc}$, which approximately corresponds to 
the size of the Milky Way Roche lobe in the Milky Way-M31 system, and $r_{\text {out }}=600 \mathrm{kpc}$, in accordance with Cox \& Loeb (2008). The particles have some distribution $\xi\left(r_{0}\right)$ over $r_{0}$ inside $\left[r_{\text {in }}, r_{\text {out }}\right]$. In accordance with recent $N$-body simulations (Stadel et al. 2009; Navarro et al. 2010), we assume that their specific angular momentum $\mu \equiv|[\boldsymbol{v} \times \boldsymbol{r}]|$ has a Gaussian distribution. Thus, the overall distribution (i.e., the mass $d m$ of particles in some interval $d r_{0} d \mu$ ) is

$$
d m=\xi\left(r_{0}\right) \frac{2 \mu}{\alpha^{2}} \exp \left(-\frac{\mu^{2}}{\alpha^{2}}\right) d \mu d r_{0}, \quad r_{0} \in\left[r_{\text {in }}, r_{\text {out }}\right],
$$

where $\alpha$ is, generally speaking, a function of $r_{0}$. We accept the envelope mass $M_{\mathrm{env}}=\int_{r_{\mathrm{in}}}^{r_{\text {out }}} \xi\left(r_{0}\right) d r_{0}=10^{12} M_{\odot}$. This value is noticeably smaller than the total mass of the diffused component, which is estimated as $\sim M_{\mathrm{MW}}+M_{31}=2.6 \times 10^{12} M_{\odot}(\mathrm{Cox}$ $\&$ Loeb 2008). We allow, however, that the main part of this substance surrounds and accretes onto the Andromeda galaxy, and takes only $M_{\mathrm{Mw}} /\left(M_{\mathrm{MW}}+M_{31}\right)$ of the total mass, so we assume that the mass should be divided proportionally to the Roche lobe areas of the components.

At first glance, it would seem that the above-stated model is completely unusable to describe the dark matter motion in the Local Group: the gravitational field of the system can by no means be considered as central on the scale $\sim 600 \mathrm{kpc}$, because of huge perturbations from other group members, especially from the Andromeda galaxy. However, we are only interested in the envelope dark matter penetration toward the solar system, and this process is totally defined by the angular momenta of the particles: only the particles with very small momenta can reach the Earth. The motion of the particles near the solar system is almost unaffected by M31 $\left(l_{\odot} \ll d\right)$ and may well be described by the above-mentioned model. When a particle moves from the envelope to the Earth, its angular momentum is, of course, strongly influenced by the tidal perturbations. However, $\mathrm{N}$-body simulations suggest that the momentum distribution of the particles remains similar to the Gaussian one down to the center of the halo (Hansen et.al. 2006; Stadel et al. 2009; Navarro et al. 2010). Therefore, Equation (1) may be thought of as describing the resultant distribution of the falling particles with regard to the perturbations from the other members of the Local Group. Moreover, as we will see, the shape of the angular momentum distribution is not very important: we actually use only the value at $\mu=0$. As for perturbations of the particle energy, they are of the order of $G M_{31} / d$, i.e., always small. This is a result of the fact that the main part of the particle acceleration takes place deep in the Milky Way, where the gravitational field is much stronger.

Now we should find the particle distribution inside radius $r_{\text {in }}$. A very similar task has been studied extensively in Baushev (2012a). We cite here only the results adaptable to our work, skipping the complete derivation. The exact distribution of the envelope dark matter density $\rho(r)$ inside $r_{\text {in }}$ depends on $r$ and is equal to

$$
\rho(r)=\int_{r_{\text {in }}}^{r_{\text {out }}} \int_{0}^{\mu_{\max }} \frac{\xi\left(r_{0}\right) r_{0} \mu \exp \left(-\mu^{2} / \alpha^{2}\right) d \mu d r_{0}}{2 \pi r \alpha^{2}\left(r_{0}\right) T\left(r_{0}, \mu\right) \sqrt{r_{0}^{2}-r^{2}} \sqrt{\mu_{\max }^{2}-\mu^{2}}} .
$$

Here, $T\left(r_{0}, \mu\right)$ is the half-period of a particle with a maximal radius $r_{0}$ and a specific angular momentum $\mu$, i.e., the time it takes for the particle to fall from its maximal radius to the minimal one, and $\mu_{\max }$ is the maximum angular momentum of a particle wherewith it can reach radius $r$ :

$$
\mu_{\max }^{2}=2\left(\phi\left(r_{0}\right)-\phi(r)\right)\left(\frac{1}{r^{2}}-\frac{1}{r_{0}^{2}}\right)^{-1} .
$$

Our concern is only with the particle distribution at $r=l_{\odot}$. Since $l_{\odot} \ll r_{\text {in }}<r_{0}$, we can simplify the above equations:

$$
\begin{gathered}
\rho=\int_{r_{\text {in }}}^{r_{\text {out }}} \int_{0}^{\mu_{\max }} \frac{\xi\left(r_{0}\right) \mu \exp \left(-\mu^{2} / \alpha^{2}\right) d \mu d r_{0}}{2 \pi l_{\odot} \alpha^{2}\left(r_{0}\right) T\left(r_{0}\right) \sqrt{\mu_{\max }^{2}-\mu^{2}}}, \\
\mu_{\max }\left(l_{\odot}\right)=l_{\odot} \sqrt{2\left(\phi\left(r_{0}\right)-\phi\left(l_{\odot}\right)\right)} .
\end{gathered}
$$

Here we took into account that for $\mu \in\left[0, \mu_{\max }\left(l_{\odot}\right)\right]$ the period $T\left(r_{0}, \mu\right)$ is almost independent of $\mu\left(T\left(r_{0}, \mu\right) \simeq T\left(r_{0}, 0\right) \equiv\right.$ $T\left(r_{0}\right)$; see Baushev 2012a for details).

Equation (4) can be significantly simplified if we take into account that $\alpha\left(r_{0}\right)$ is physically constrained. On one hand, $\alpha\left(r_{\text {out }}\right)$ can hardly be higher than

$$
\alpha\left(r_{\text {out }}\right)=\frac{1}{3} r_{\text {out }} \sqrt{\frac{2 G\left(M_{\mathrm{MW}}+M_{\mathrm{env}}\right)}{r_{\mathrm{out}}}}
$$

since in the opposite case a significant fraction of the particles would have a speed above the escape velocity at this radius. On the other hand, numerical simulations (Stadel et al. 2009) show that the root-mean-square angular momentum of the particles should be quite high and close to the upper limit (6). Though there is rather strong evidence that $\alpha$ of the particles of our Galaxy is much lower (Baushev 2011), we will use the value (6) in our calculations, since the density of the extragalactic dark matter grows with decreasing $\alpha$, and we take the maximum possible value in order to obtain a conservative estimate. The dependence of $\alpha$ on $r_{0}$ is not well known; $N$-body simulations suggest $\alpha\left(r_{0}\right) \propto r_{0}$ (Macciò et al. 2007). For generality, we will presume the dependence to be power law:

$$
\alpha\left(r_{0}\right)=\alpha\left(r_{\text {out }}\right)\left(\frac{r_{0}}{r_{\text {out }}}\right)^{i} .
$$

However, we only need a much softer condition $\alpha\left(r_{0}\right) \geqslant$ $\mu_{\max }\left(l_{\odot}\right)$ to simplify Equation (4). This means that the tangential velocity dispersion in the envelope is supposed to be higher than $v_{\text {esc }}\left(l_{\odot} / r_{\text {in }}\right) \simeq 16 \mathrm{~km} \mathrm{~s}^{-1}$. Such an assumption seems quite natural. If $\alpha\left(r_{0}\right) \geqslant \mu_{\max }\left(l_{\odot}\right)$, then we can simplify Equation (4) as

$$
\rho=\int_{r_{\text {in }}}^{r_{\text {out }}} \int_{0}^{\mu_{\max }} \frac{\xi\left(r_{0}\right) \mu d \mu d r_{0}}{2 \pi l_{\odot} \alpha^{2}\left(r_{0}\right) T\left(r_{0}\right) \sqrt{\mu_{\max }^{2}-\mu^{2}}} .
$$

Now we should ascertain the velocity distribution of the particles. Let us denote the tangential and radial components, and the total velocity of a particle at $r=l_{\odot}$ by $u_{\tau}, u_{r}$, and $u$, respectively. We also define an angle $\theta$ between the particle velocity and the direction toward the Galaxy center: $\sin \theta=u_{\tau} / u$. Since $\mu=u_{\tau} l_{\odot}$ and $\mu_{\max }=u l_{\odot}$, we have $\sin \theta=\mu / \mu_{\max }$. The particle velocity distribution function $f$ (in the frame of reference that does not rotate around the Galaxy center) can depend on only two parameters, $u$ and $\theta$, in view of the spherical symmetry of the system:

$$
\rho=\int_{u=0}^{\infty} \int_{\theta=0}^{\pi} f(u, \theta) u^{2} d u d \Omega .
$$


In order to find the angular distribution, we should replace the coordinates $\left(\mu, r_{0}\right)$ in Equation (8) with $(u, \theta)$. However, $u$ and $r_{0}$ are bound by a one-to-one relation:

$$
u=\sqrt{2\left(\phi\left(r_{0}\right)-\phi\left(l_{\odot}\right)\right)}, \quad d u=\frac{\left(\frac{d \phi\left(r_{0}\right)}{d r_{0}}\right) d r_{0}}{\sqrt{2\left(\phi\left(r_{0}\right)-\phi\left(l_{\odot}\right)\right)}}
$$

and we can use coordinates $\left(r_{0}, \theta\right)$ in order to find the angular distribution. An element $d \Omega$ of the solid angle in the phase space is equal to

$$
d \Omega=\frac{u}{u_{r}} \frac{2 \pi u_{\tau} d u_{\tau}}{4 \pi u^{2}}=\frac{\mu d \mu}{2 l_{\odot} \sqrt{\mu_{\max }^{2}-\mu^{2}} \sqrt{2\left(\phi\left(r_{0}\right)-\phi\left(l_{\odot}\right)\right)}} .
$$

Substituting this equation into Equation (8), we obtain

$$
\rho=\int_{r_{\text {in }}}^{r_{\text {out }}} \int \frac{\xi\left(r_{0}\right) \sqrt{2\left(\phi\left(r_{0}\right)-\phi\left(l_{\odot}\right)\right)} d r_{0}}{\pi \alpha^{2}\left(r_{0}\right) T\left(r_{0}\right)} d \Omega .
$$

Comparing Equations (8) and (9) and using the relationships in (10), we obtain

$$
f(u, \theta)=\frac{\xi\left(r_{0}\right)}{\pi \alpha^{2}\left(r_{0}\right) T\left(r_{0}\right)\left(\frac{d \phi\left(r_{0}\right)}{d r_{0}}\right)} .
$$

As we can see, particle distribution $f$ depends only on $r_{0}$ (i.e., only on velocity magnitude $u$; see Equation (10)), and is independent of $\theta$. Hence the velocity distribution of the dark matter from the envelope is isotropic near the solar system. Then we can integrate Equation (12) over $d \Omega\left(\int d \Omega=4 \pi\right)$ :

$$
\rho=\int_{\sqrt{2\left(\phi\left(r_{\text {in }}\right)-\phi\left(l_{\odot}\right)\right)}}^{\sqrt{2\left(\phi\left(r_{\text {out }}\right)-\phi\left(l_{\odot}\right)\right)}} \frac{8 \xi\left(r_{0}\right)\left(\phi\left(r_{0}\right)-\phi\left(l_{\odot}\right)\right)}{\alpha^{2}\left(r_{0}\right) T\left(r_{0}\right)\left(d \phi\left(r_{0}\right) / d r_{0}\right)} d u .
$$

In order to complete the solution, we should define functions $M(r), \phi\left(r_{0}\right), T\left(r_{0}\right)$, and $\xi\left(r_{0}\right)$. Unfortunately, now we have no reliable experimental data about the mass distribution in the envelope. $N$-body simulations suggest, however, that the density profile may be a power law (Navarro et al. 1997, 2010; Cox \& Loeb 2008; Stadel et al. 2009). We will assume that the mass distribution at $r_{\text {out }}>r>r_{\text {in }}$ may be represented (or, at least, properly approximated) by a power-law dependence with some index $j$ :

$$
M(r) \simeq M_{\mathrm{MW}}+M_{\mathrm{env}}\left(\frac{r-r_{\mathrm{in}}}{r_{\mathrm{out}}-r_{\mathrm{in}}}\right)^{j+1}
$$

The function $\left(d \phi\left(r_{0}\right) / d r_{0}\right)=\left(G M\left(r_{0}\right) / r_{0}^{2}\right)$ is totally defined by Equation (15):

$$
\frac{d \phi\left(r_{0}\right)}{d r_{0}}=\frac{G}{r_{0}^{2}}\left[M_{\mathrm{MW}}+M_{\mathrm{env}}\left(\frac{r_{0}-r_{\mathrm{in}}}{r_{\mathrm{out}}-r_{\mathrm{in}}}\right)^{j+1}\right] .
$$

Integrating Equation (1) over $\mu$, we determine that the mass of the particles with apoapses in an interval $d r$ is $\xi(r) d r$. It is natural to assume that $(d M / d r)=\xi(r)$. Differentiating Equation (15), we obtain

$$
\xi\left(r_{0}\right)=\frac{(j+1) M_{\mathrm{env}}}{r_{\mathrm{out}}-r_{\mathrm{in}}}\left(\frac{r_{0}}{r_{\mathrm{out}}-r_{\mathrm{in}}}\right)^{j} .
$$

The half-period $T\left(r_{0}\right)$ of a particle with a maximal radius $r_{0}$ and zero specific angular momentum is

$$
T\left(r_{0}\right)=\int_{0}^{r_{0}} \frac{d r}{v_{r}}=\int_{0}^{r_{0}} \frac{d r}{\sqrt{2\left[\phi(r)-\phi\left(r_{0}\right)\right]}} .
$$

In order to find $T\left(r_{0}\right)$, we should integrate Equation (16) and then substitute the obtained gravitational potential $\phi(r)$ into Equation (18). However, distribution (15) is rather complex, and the analytical solution of the task is hardly possible. On the other hand, there is a much simpler way to obtain a good approximation. First of all, $T\left(r_{\text {in }}\right) \simeq 10^{17} \mathrm{~s}$, which is approximately the time necessary for a body to fall on point mass $M_{\mathrm{MW}}=10^{12} M_{\odot}$ from $300 \mathrm{kpc}$ with no initial velocity. Second, since the ratio $r_{\text {out }} / r_{\text {in }}$ is relatively small in our case, we can approximate $T\left(r_{0}\right)$ by a power-law function. The index of power weakly depends on the mass distribution: $T\left(r_{0}\right) \propto r_{0}$ for isothermal profile $\rho \propto r^{-2}$ (Baushev 2011) and $T\left(r_{0}\right) \propto r_{0}^{3 / 2}$ for the gravitational field of a point mass. The distinction in $T\left(r_{0}\right)$ for these two completely different mass distributions does not exceed $\sqrt{2}$. Meanwhile, any realistic density profile of the envelope is steeper than $\rho \propto r^{-2}$ for $r \in\left[r_{\text {in }}, r_{\text {out }}\right]$ (Navarro et al. 1997; Einasto 1969) and shallower than the point mass. It is easy to see that $T\left(r_{0}\right) \propto r^{1-\frac{j}{2}}$ if $M(r) \propto r^{j+1}$, and we can assume that the index of power is the same for distribution (16) if $r_{0} \in\left[r_{\text {in }}, r_{\text {out }}\right]$. We obtain

$$
T\left(r_{0}\right)=T\left(r_{\text {in }}\right)\left(\frac{r_{0}}{r_{\text {in }}}\right)^{1-\frac{j}{2}}
$$

and $T\left(r_{\text {in }}\right)=10^{17} \mathrm{~s}$. In order to make a good approximation of the function $\sqrt{2\left(\phi\left(r_{0}\right)-\phi\left(l_{\odot}\right)\right)}$, we should estimate potentials $\phi\left(r_{0}\right)$ and $\phi\left(l_{\odot}\right) . \phi\left(l_{\odot}\right)=-v_{\text {esc }}^{2} / 2$ is defined by the galactic escape speed near the solar system $v_{\text {esc }}$. $v_{\text {esc }}$ depends on the matter distribution in the Local Group and now is not exactly known; it undoubtedly exceeds $525 \mathrm{~km} \mathrm{~s}^{-1}$ (Carney \& Latham $1987)$ and in principle may be much larger $\left(650 \mathrm{~km} \mathrm{~s}^{-1}\right.$ or even higher; Binney \& Tremaine 2008; Marochnik \& Suchkov 1984), i.e., $\left|\phi\left(l_{\odot}\right)\right|>1.3 \times 10^{5} \mathrm{~km}^{2} \mathrm{~s}^{-2}$. On the other hand, $\left|\phi\left(r_{\text {out }}\right)\right| \simeq G\left(M_{\text {env }}+M_{\mathrm{MW}}\right) / r_{\text {out }} \simeq 1.4 \times 10^{4} \mathrm{~km}^{2} \mathrm{~s}^{-2}$ and $\left|\phi\left(r_{\text {out }}\right)-\phi\left(r_{\text {in }}\right)\right| \simeq G M_{\mathrm{MW}}\left(r_{\text {out }}-r_{\text {in }}\right) / r_{\text {in }}^{2} \simeq 1.4 \times 10^{4} \mathrm{~km}^{2} \mathrm{~s}^{-2}$. Consequently, the function $\sqrt{2\left(\phi\left(r_{0}\right)-\phi\left(l_{\odot}\right)\right)}$ remains almost constant for $r_{0} \in\left[r_{\text {in }} ; r_{\text {out }}\right]$ owing to the smallness of $\mid \phi\left(r_{\text {out }}\right)-$ $\phi\left(r_{\text {in }}\right) \mid$ as compared with $\left|\phi\left(r_{\text {in }}\right)-\phi\left(r_{l_{\odot}}\right)\right|$. Therefore, we can approximate $\sqrt{2\left(\phi\left(r_{0}\right)-\phi\left(l_{\odot}\right)\right)} \simeq \sqrt{2\left(\phi\left(r_{\text {in }}\right)-\phi\left(l_{\odot}\right)\right)} \equiv V$. It is easy to see that $\left(v_{\text {esc }}-V\right) / v_{\text {esc }} \simeq \phi\left(r_{\text {out }}\right) /\left(2 \phi\left(l_{\odot}\right)\right) \simeq 5 \%$, i.e., $V$ should be very close to the galactic escape speed near the solar system. In accordance with the above-mentioned estimations of $v_{\text {esc }}$, we accept $V=600 \mathrm{~km} \mathrm{~s}^{-1}$. The velocities of all the particles lie in a very narrow interval $\Delta V$ :

$$
\Delta V=\sqrt{V^{2}+2\left(\phi\left(r_{\text {out }}\right)-\phi\left(r_{\text {in }}\right)\right)}-V .
$$

Now we should substitute Equations (7), (19), and (17) for $\alpha\left(r_{0}\right)$, $T\left(r_{0}\right)$, and $\xi\left(r_{0}\right)$, respectively, and Equation (6) for $\alpha\left(r_{\text {out }}\right)$ into Equation (14). It is convenient to introduce $k \equiv r_{\text {out }} / r_{\text {in }}=2$. After some trivial calculations, we obtain

$$
\rho=9 \frac{(j+1)}{\left(\frac{3}{2} j-2 i\right)} \frac{k^{2 j}\left(k^{\frac{3}{2} j-2 i}-1\right)}{(k-1)^{j+1}} \frac{V}{G T\left(r_{\text {in }}\right) r_{\text {out }}} .
$$




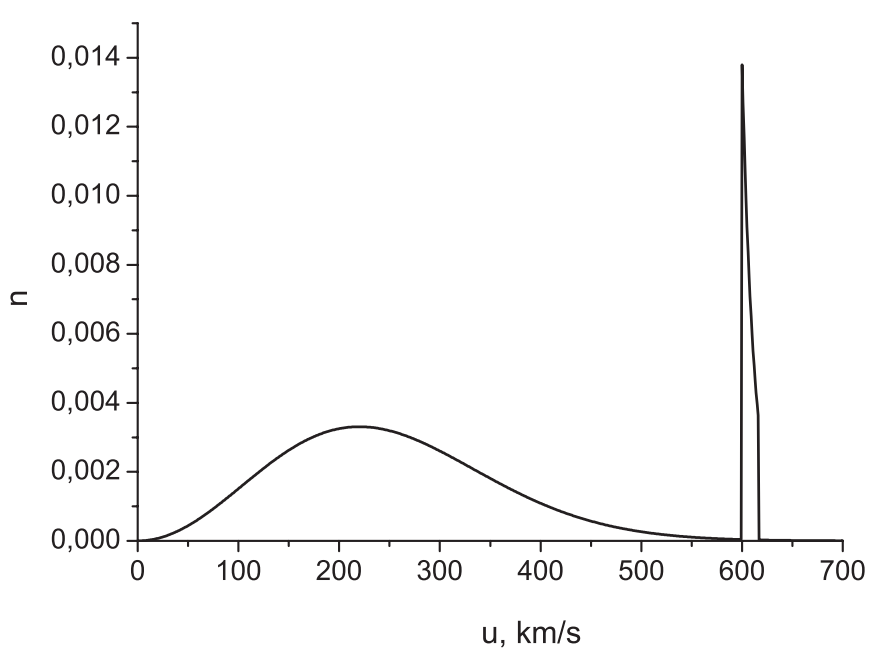

Figure 1. Normalized velocity distribution of DMPs near the Earth in the frame of reference that does not rotate around the Galaxy center. The distribution of the galactic DMPs is supposed to be Maxwell (26). The extragalactic component gives a narrow high peak near $600 \mathrm{~km} \mathrm{~s}^{-1}$.

The shape of the velocity distribution function $n$ in the phase space is given by

$$
n(u) \propto \frac{d u^{3}}{4 \pi u^{2}} r_{0}^{\frac{3}{2} j-2 i-1}\left(\frac{d r_{0}}{d u}\right),
$$

where we should substitute $r_{0}$ by $u \in[V, V+\Delta V]$ with the help of Equations (17) and (16). Equations (21) and (22) completely determine the solution of the task.

\section{RESULTS AND DISCUSSION}

Little is known about the dark matter distribution in the envelope. On the other hand, as we can see from Equation (21), the result is not strongly dependent on the choice of $i$ and $j$ (as a consequence of the relatively small ratio $\left.r_{\text {out }} / r_{\text {in }}\right)$. It seems reasonable to choose $i$ and $j$ by analogy with the well-known isothermal halo solution $(d M / d r=$ const and the Maxwell DMP velocity distribution with a temperature, constant over the halo), which corresponds to $i=1, j=0$. Substituting these values in combination with $T\left(r_{\text {in }}\right), \alpha\left(r_{\text {in }}\right)$, and $V$ into Equation (21), we obtain the density of the extragalactic dark matter near the Earth as $\rho=3.7 \times 10^{-2} \mathrm{GeV} \mathrm{cm}^{-3}$.

Figure 1 shows that the speed distribution is notably narrow: the absolute values of all particles fall within $\Delta V \simeq 16 \mathrm{~km} \mathrm{~s}^{-1}$. As we have already mentioned, this feature is a consequence of the small value of $\left|\phi\left(r_{\text {out }}\right)-\phi\left(r_{\text {in }}\right)\right|$ as compared with $-\phi\left(l_{\odot}\right)$. Therefore, two properties of the velocity distribution are model-independent: the speeds of extragalactic DMPs from the envelope lie in a narrow range, and their angular distribution is isotropic.

The density of the extragalactic dark matter turns out to be fairly high: $3.7 \times 10^{-2} \mathrm{GeV} \mathrm{cm} \mathrm{cm}^{-3}$ is more than $12 \%$ of the total dark matter density near the Earth $\simeq 0.3 \mathrm{GeV} \mathrm{cm}^{-3}$ (Gorbunov \& Rubakov 2011). This brings up a question: how reliable is the estimation? Above, we have already discussed the approximation of the system by a spherically symmetric model and found it acceptable. The premise that $\xi\left(r_{0}\right)$ terminates abruptly at $r_{\text {in }}$ and $r_{\text {out }}$ is also unphysical. Undoubtedly, our result is assessed; however, it cannot be called optimistic. Indeed, as the dimensional method shows, for any envelope model the density of the extragalactic dark matter is, with an accuracy of a numerical factor, equal to

$$
\rho \propto \frac{M_{\mathrm{env}} v_{\mathrm{esc}}}{\langle\alpha\rangle^{2}\langle T\rangle}
$$

where $\langle\alpha\rangle$ and $\langle T\rangle$ are the average values of the respective quantities. Our calculations confirm this dependence: it can be easily obtained from Equation (14). $v_{\text {esc }}$ is almost independent of the model choice. $\langle T\rangle$ is essentially defined by the size of the Milky Way Roche lobe, and thus is also more or less modelindependent. The main source of the uncertainty is the envelope mass $M_{\text {env }}$. We proceed from the assumption of Cox \& Loeb (2008) that $M_{\text {env }}$ is approximately equal to the masses of the galaxies of the Local Group. We used the highest possible value (6) for $\alpha$; if $\alpha$ was higher, then a significant part of the envelope would rapidly evaporate. Since $\rho \propto \alpha^{-2}$, this choice is conservative.

Thus there are two possible situations when Equation (21) significantly overestimates the density of the extragalactic dark matter. It may be so if the envelope mass is in fact much lower than the masses of the Local Group member galaxies. The strong overestimation may also appear if the angular momentum distribution of the envelope DMPs differs greatly from the Gaussian (1), i.e., almost all the particles have circular orbits. Such a supposition seems highly improbable. First, it is in sharp contrast to $N$-body simulation results (Stadel et al. 2009). There is also a good indirect counterargument: the largest Local Group member, M31, has quite low angular momentum and, consequently, a very oblong orbit (Kahn \& Woltjer 1959). It is plausible that the Milky Way and M31 will finally experience a central collision. Thus, the presence in the diffuse component of the Local Group of a bulk of DMPs that have very oblong orbits and can reach the Earth seems quite possible.

The 12\% extragalactic component of the total dark matter density can be especially important for the direct dark matter search. The direct search is based on the detection of the collisions of DMPs with nuclei of the target. The signal is sensitive to the velocity distribution; roughly speaking (Bélanger et al. 2009), it is proportional to

$$
I\left(v_{\min }\right)=\int_{v_{\min }}^{\infty} v \tilde{f}(v) d v .
$$

Here, $\tilde{f}(v)$ is the distribution over the velocity magnitude in the Earth's frame of reference: it should be obtained from Equation (22), (26), or (27) by a Galilean transformation and an integration over all the directions. $v_{\min }$ is the minimal DMP speed to which the detector is sensitive (for details, see Bélanger et al. 2009):

$$
v_{\min }^{2} \simeq \frac{E_{A}}{2} \frac{\left(m_{\chi}+m_{A}\right)^{2}}{m_{A} m_{\chi}^{2}},
$$

where $m_{\chi}$ and $m_{A}$ are the DMP and the detector nucleus mass, respectively, and $E_{A}$ is the detector activation energy, depending on its construction. In order to estimate the influence of the extragalactic dark matter to the direct detection signal, we should know the velocity distribution of the galactic component. Unfortunately, we are still a long way from solving the problem. All the direct detection experiments routinely use the Maxwell distribution:

$$
f(u)=\frac{1}{\left(\sqrt{\pi} v_{\odot}\right)^{3}} \exp \left(-\frac{u^{2}}{v_{\odot}^{2}}\right),
$$




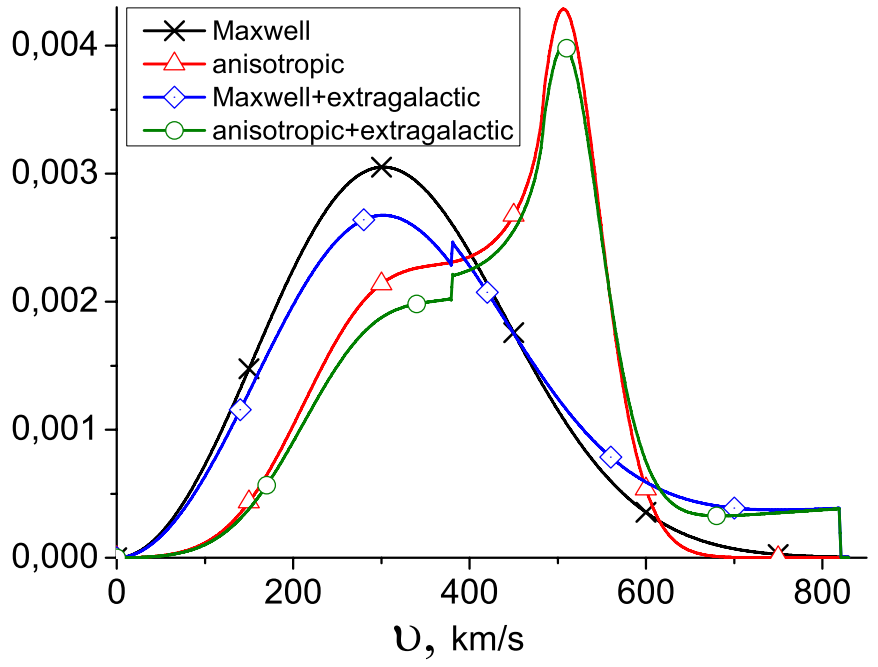

Figure 2. Velocity magnitude distributions in the Earth's frame of reference for four models: Maxwell (26) (line with crosses), anisotropic (27) (line with triangles), $\sim 12.3 \%$ of extragalactic component $(21)$ and $87.7 \%$ of the Maxwell galactic DMPs (26) (line with diamonds), and $\sim 12.3 \%$ of extragalactic component (21) and $87.7 \%$ of the anisotropic galactic DMPs (27) (line with circles). All the distributions are normalized to 1 .

(A color version of this figure is available in the online journal.)

where $v_{\odot}$ is the orbital speed of the solar system. Besides simplicity, the Maxwell distribution has two more solid arguments in its favor. First, the usage of the same distribution allows us to directly compare the results of different experiments. Second, Equation (26) is an exact solution for an isothermal halo $\left(\rho \propto r^{-2}\right)$, under the assumption that the distribution is isotropic. We should emphasize that the Maxwell distribution is a solution only for an infinite isothermal halo (with an infinite mass) and is not valid for a realistic one.

However, the real velocity distribution can hardly be Maxwell. First, it is unclear how the Maxwell distribution could appear in the collisionless system. Second, $N$-body simulations (Vergados et al. 2008; Kuhlen et al. 2010) suggest that the distribution is anisotropic with $\sigma\left(v_{r}\right)>\sigma\left(v_{\tau}\right)$. The later relationship is indirectly confirmed by the fact that the motion of the stellar halo objects (which was probably formed simultaneously with the dark one) is also almost radial (Baushev 2011). Finally, the streams created by the tidal destruction of small halos can strongly influence the velocity distribution function. For instance, Purcell et al. (2012) showed that the Sagittarius stream alone can make a 2\%-3\% contribution to the total dark matter density near the solar system and $\sim 10 \%$ contribution to the direct detection signal. Of course, such an impact is also very important.

In order to estimate the influence of the extragalactic component on the direct detection signal and be more or less independent of the uncertainties of the galactic distribution model, we will consider two extreme cases. As we have already mentioned, the Maxwell distribution (26) gives an exact analytical solution for the infinite isothermal profile $\rho \propto r^{-2}$, if we suppose the velocity distribution to be isotropic. It turns out that in the opposite case of an extremely anisotropic distribution, an exact analytical solution for a finite isothermal halo can be found as well (Baushev 2011). We will use this model as the very anisotropic case:

$$
f(u)=\frac{\exp \left(-\frac{u_{\tau}^{2}}{2 \sigma_{0}^{2}}\right)}{2 \pi^{2} \sigma_{0}^{2} \sqrt{u_{\max }^{2}-u_{r}^{2}}},
$$

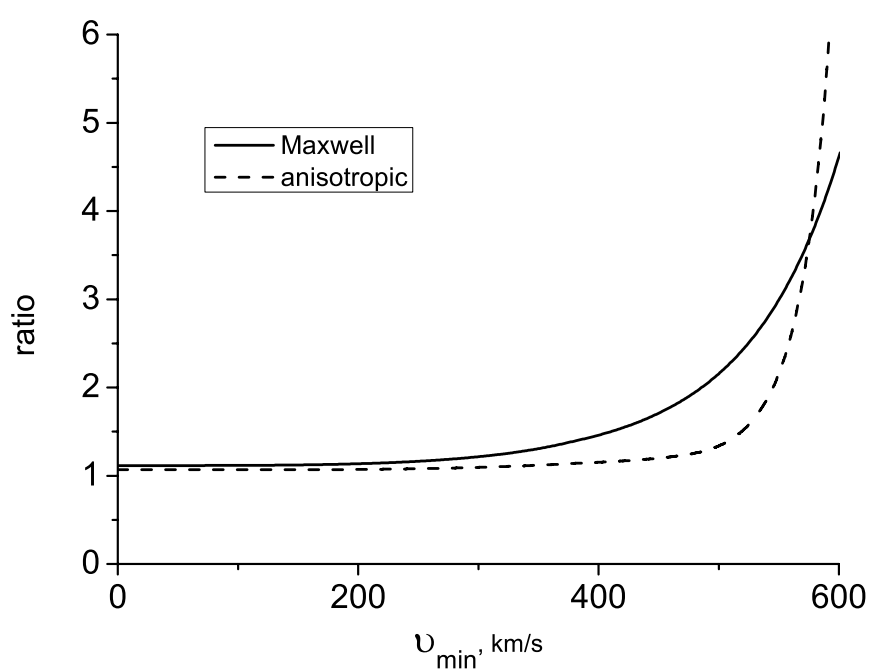

Figure 3. Ratio of the direct detection signal produced by the mixture $\sim 12.3 \%$ of extragalactic component and $87.7 \%$ of the galactic DMPs to the signal produced by pure galactic dark matter. The fraction and velocity distribution of the extragalactic component were calculated in accordance with Equation (21). We considered two models of the velocity distribution of the galactic DMPs: Maxwell (26) (solid line) and anisotropic (27) (dashed line). The extragalactic dark matter almost does not affect the signal, if $v_{\min }<300 \mathrm{~km} \mathrm{~s}^{-1}$, but totally dominates above $450-500 \mathrm{~km} \mathrm{~s}^{-1}$.

where $u_{r} \in\left[-v_{\max } ; v_{\max }\right], u_{\max } \simeq 560 \mathrm{~km} \mathrm{~s}^{-1}, \sigma_{0}=80 \mathrm{~km} \mathrm{~s}^{-1}$, and $u_{r}$ and $u_{\tau}$ are the radial and tangential components of the particle velocity, respectively. We may expect that any reasonable galactic component distribution lies between the extreme cases (26) and (27), though this statement is not quite correct if we consider the annual modulations of the signal. Our calculations show no significant difference in the shape of the annual modulations of the extragalactic signal with respect to that produced by distribution (26) or (27). However, distributions (26) and (27) and distribution (22) of the extragalactic component are symmetric in the sense that they depend only on the absolute value of the tangential velocity component $u_{\tau}$. The streams created by the small halos, such as that of Sagittarius (Purcell et al. 2012), may strongly violate this symmetry. Thus the annual modulations produced by the extragalactic component should coincide with those predicted by the conventional Maxwell distribution, but may be shifted with respect to those produced by the real galactic component. Figure 2 represents the velocity magnitude distributions in the Earth's frame of reference for four models: pure Maxwell (26), pure anisotropic (27), $\sim 12.3 \%$ of extragalactic component (21) and $87.7 \%$ of the Maxwell galactic DMPs (26), and $\sim 12.3 \%$ of extragalactic component (21) and $87.7 \%$ of the anisotropic galactic DMPs (27).

We calculated the signal produced by a mixture of $3.7 \times 10^{-2} \mathrm{GeV} \mathrm{cm}^{-3}$ of the extragalactic dark matter and $0.263 \mathrm{GeV} \mathrm{cm}^{-3}$ of the galactic one and divided it by the signal produced by the pure galactic dark matter with the same total density $\left(0.3 \mathrm{GeV} \mathrm{cm}^{-3}\right)$. We used both models of the galactic DMP distribution: Maxwell (26) and anisotropic (27). The two ratios are represented in Figure 3 by the solid line (Maxwell velocity distribution of the galactic DMPs) and by the dashed line (anisotropic velocity distribution). One can see that the signal is scarcely affected by the presence of the extragalactic component if $v_{\max }<300 \mathrm{~km} \mathrm{~s}^{-1}$. However, the situation drastically changes for higher $v_{\max }$; if $v_{\max }$ is larger than $450-500 \mathrm{~km} \mathrm{~s}^{-1}$, then the extragalactic signal dominates. This is not particularly surprising: all the extragalactic particles are faster than 
$600 \mathrm{~km} \mathrm{~s}^{-1}$, while the number of the galactic particles rapidly drops above $\sim 450 \mathrm{~km} \mathrm{~s}^{-1}$. Hence the impact of the extragalactic dark matter on the direct detection signal can be very important, especially if the DMP mass $m_{\chi}$ is small. Indeed, if $m_{\chi}$ is small, then $v_{\max }$ is high (25), i.e., we can detect only the fastest DMPs. The DAMA Collaboration (Bernabei et al. 2011) detected a signal that might be produced by $\sim 10 \mathrm{GeV}$ weakly interacting massive particles (WIMPs). Several other direct detection experiments also reported some hints of $\sim 10 \mathrm{GeV}$ WIMP presence (for instance, the very late CDMS observations revealed three WIMP-candidate events, corresponding to a $\sim 8.6 \mathrm{GeV}$ WIMP with $\sim 1.9 \times 10^{-41} \mathrm{~cm}^{2}$ WIMP-nucleon cross section; CDMS Collaboration et al. 2013). We shall not discuss here the question of the nature of the signals (the situation is quite complex and controversial, and other detectors do not confirm the result; Aprile et al. 2011). It should be noted, however, that $v_{\max }>450 \mathrm{~km} \mathrm{~s}^{-1}$ for the majority of detectors if the DMP is so light, i.e., the extragalactic component signal should dominate. For instance, the $v_{\min }$ threshold is, roughly speaking, equal to 300,400 , and $500 \mathrm{~km} \mathrm{~s}^{-1}$ for the CoGeNT, DAMA, and CRESS experiments, respectively, if we accept the DMP mass of $10 \mathrm{GeV}$ (Fox et al. 2011). The extragalactic component gives $28 \%$ of the total direct detection signal if the distribution of the galactic component is Maxwell (26), and 20\% if it is anisotropic (27) for $v_{\min }=300 \mathrm{~km} \mathrm{~s}^{-1}, 40 \%$ and $23 \%$ for $v_{\min }=400 \mathrm{~km} \mathrm{~s}^{-1}$, and $59 \%$ and $34 \%$ for $v_{\min }=500 \mathrm{~km} \mathrm{~s}^{-1}$.

Note that we should, strictly speaking, have cut distributions (26) and (27) at $u=v_{\text {esc }}$. However, the fraction of particles with $u>v_{\text {esc }}$ is negligible for both distributions, and cutting them would hardly affect the result; the impact of the extragalactic component would be even slightly higher.

We performed our calculations under the assumption that the dark matter density near the solar system is $0.3 \mathrm{GeV} \mathrm{cm}^{-3}$. This value is conventional; however, it continues to be challenged and probably will be revised upward. For instance, Garbari et al. (2012) found a local dark matter density of $0.95 \pm 0.5 \mathrm{GeV} \mathrm{cm}{ }^{-3}$. Recent simulations by Purcell et al. (2012) also suggest a local dark matter density of $0.5-0.6 \mathrm{GeV} \mathrm{cm}^{-3}$. If the galactic component density is higher, then the fraction of the signal produced by the extragalactic component diminishes. On the other hand, our estimation of the density of the extragalactic component is also approximate. Figure 4 illustrates both these effects. The gray and hatched bands represent the possible fraction of the direct detection signal produced by the extragalactic component, depending on the local density of the galactic component. We accept $v_{\min }=500 \mathrm{~km} \mathrm{~s}^{-1}$, and the upper and lower borders of each band correspond to galactic component distributions (26) and (27), respectively. The hatched band represents the above-considered case of the envelope model with $i=1$ and $j=0$, and the gray one the envelope with $i=1$ and $j=1$. As we expected, the impact of the extragalactic component diminishes as the dark matter density grows. However, the impact can still be significant even for high densities.

In conclusion, let us briefly consider the extragalactic dark matter that does not belong to the Local Group. As recent astronomical observations imply (Makarov \& Karachentsev 2011), the dark matter of the Virgo Supercluster, in addition to galaxies and their groups, forms a large diffuse component. We do not know its velocity and space distributions, but it seems reasonable to assume that the dark matter is distributed more or less uniformly, and the velocity dispersion of the DMPs is comparable with that of the observable members

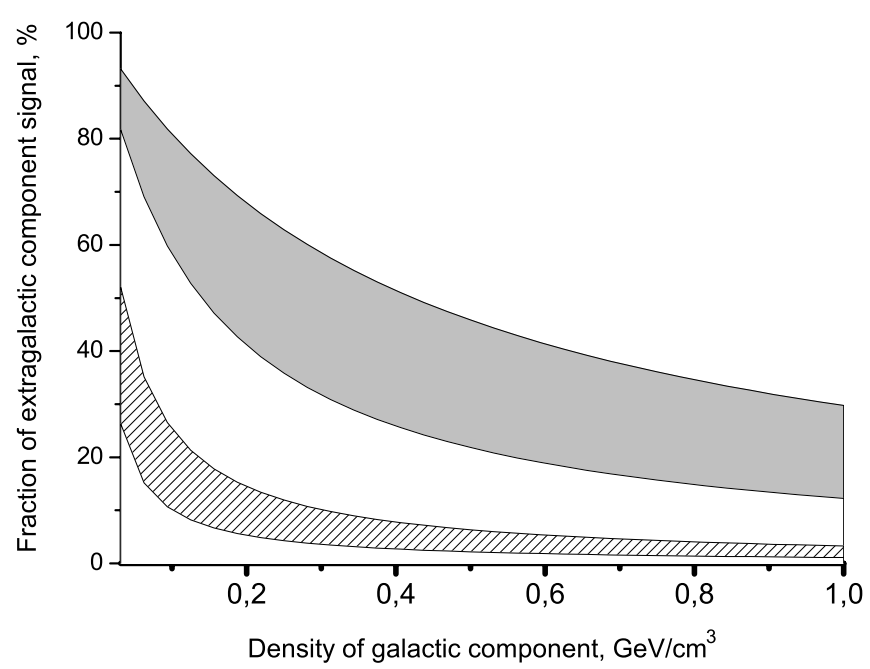

Figure 4. Fraction of direct detection signal produced by the extragalactic component, depending on the local density of the galactic component. The hatched band represents the above-considered case of the envelope model with $i=1, j=0$, and the gray one the envelope with $i=1, j=1$. We accept $v_{\min }=500 \mathrm{~km} \mathrm{~s}^{-1}$, the upper and lower borders of each band correspond to galactic component distributions (26) and (27), respectively.

of the Supercluster $\left(v_{\infty} \sim 500 \mathrm{~km} \mathrm{~s}^{-1}\right)$. The measurements estimate the average density of the diffuse component as

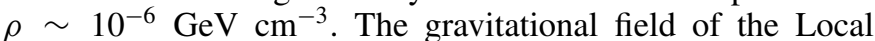
Group should increase this quantity near the Earth. However, we can roughly estimate the enhancement as $1+v_{\text {esc }}^{2} / v_{\infty}^{2}$, where $v_{\text {esc }} \simeq 650 \mathrm{~km} \mathrm{~s}^{-1}$ is the escape velocity from the solar system orbit (Baushev 2012b). Thus, the density of the Supercluster dark matter is approximately three times higher near the solar system, but hardly exceeds $10^{-5} \mathrm{GeV} \mathrm{cm}^{-3}$. This value is so low that it may scarcely be of interest for modern experiments. On the other hand, the Supercluster DMPs are particularly energetic $\left(v>1000 \mathrm{~km} \mathrm{~s}^{-1}\right)$, and hence may give a very characteristic signal.

Finally, the results are summarized as follows.

1. The particles of the diffuse component of the Local Group are apt to contribute $\gtrsim 10 \%$ to the total dark matter density near the Earth.

2. The particle speeds are $\sim 600 \mathrm{~km} \mathrm{~s}^{-1}$, i.e., they are much faster than the galactic DMPs. The particles have an isotropic velocity distribution (perhaps in contrast to the galactic dark matter); their speed distribution is very narrow $\left(\Delta V \sim 20 \mathrm{~km} \mathrm{~s}^{-1}\right)$.

3. The extragalactic dark matter should give a significant contribution to the direct detection signal. If the detector is sensitive only to the fast particles $\left(v>450 \mathrm{~km} \mathrm{~s}^{-1}\right)$, then the signal may even dominate.

4. The density of other types of extragalactic dark matter (for instance, of the DMPs forming the diffuse component of the Virgo Supercluster) should be relatively small and comparable with the average dark matter density of the universe. However, these particles can generate anomaly high-energy collisions in direct dark matter detectors.

Financial support by the Bundesministerium für Bildung und Forschung through DESY-PT, grant 05A11IPA, is gratefully acknowledged. BMBF assumes no responsibility for the contents of this publication. We acknowledge support by the Helmholtz Alliance for Astroparticle Physics HAP funded by the Initiative and Networking Fund of the Helmholtz Association. 


\section{REFERENCES}

Aprile, E., Arisaka, K., Arneodo, F., et al. (XENON100 Collaboration) 2011, PhRvL, 107, 131302

Baushev, A. N. 2011, MNRAS, 417, L83

Baushev, A. N. 2012a, arXiv:1205.4302

Baushev, A. N. 2012b, MNRAS, 420, 590

Bélanger, G., Nezri, E., \& Pukhov, A. 2009, PhRvD, 79, 015008

Bernabei, R., Belli, P., di Marco, A., et al. 2011, in AIP Conf. Proc. 1417, Workshop on Calculation of Double-beta-decay Matrix Elements, ed. O. Civitarese, I. Stekl, \& Jouni Suhonen (Melville, NY: AIP), 12

Binney, J., \& Tremaine, S. 2008, Galactic Dynamics (2nd ed.; Princeton, NJ: Princeton Univ. Press)

Carney, B. W., \& Latham, D. W. 1987, in IAU Symp. 117, Dark Matter in the Universe, (Dordrecht: Reidel), 39

CDMS Collaboration, Agnese, R., Ahmed, Z., et al. 2013, arXiv: 1304.4279

Cox, T. J., \& Loeb, A. 2008, MNRAS, 386, 461
Einasto, J. 1969, Afz, 5, 137

Fox, P. J., Liu, J., \& Weiner, N. 2011, PhRvD, 83, 103514

Garbari, S., Liu, C., Read, J. I., \& Lake, G. 2012, MNRAS, 425, 1445

Gorbunov, D. S., \& Rubakov, V. A. 2011, Hot Big Bang Theory, Vol. 1 (Singapore: World Scientific)

Hansen, S. H., Moore, B., Zemp, M., \& Stadel, J. 2006, JCAP, 01, 014

Kahn, F. D., \& Woltjer, L. 1959, ApJ, 130, 705

Kuhlen, M., Weiner, N., Diemand, J., et al. 2010, JCAP, 02, 030

Macciò, A. V., Dutton, A. A., van den Bosch, F. C., et al. 2007, MNRAS, 378,55

Makarov, D., \& Karachentsev, I. 2011, MNRAS, 412, 2498

Marochnik, L. S., \& Suchkov, A. A. 1984, The Galaxy (Moscow: Izdatel'stvo Nauka), in Russian

Navarro, J. F., Frenk, C. S., \& White, S. D. M. 1997, ApJ, 490, 493

Navarro, J. F., Ludlow, A., Springel, V., et al. 2010, MNRAS, 402, 21

Purcell, C. W., Zentner, A. R., \& Wang, M.-Y. 2012, JCAP, 08, 027

Stadel, J., Potter, D., Moore, B., et al. 2009, MNRAS, 398, L21

Vergados, J. D., Hansen, S. H., \& Host, O. 2008, PhRvD, 77, 023509 